1

$\frac{y}{\text { fy. }} \frac{1}{\text { code }} \operatorname{cet} 19 \frac{\text { Semiannual status Report }}{\text { NASA Grant 05-010-008 }}$

$N 6 I_{\text {I. }}^{19035}$

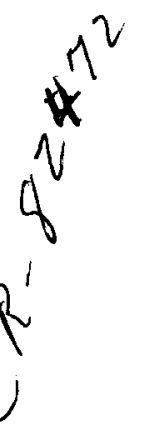

1. I had done some work on the reflection laws of systems of elliptic equations across a straight line on which general analytic linear boundary conditions are given. While working on this grant last summer, I extended these results, in the enclosed paper "Reflection Laws of Systems of Second Order Elliptic Differential Equations in Two Independent Variables with Constant Coefficients", to reflection across a general analytic arc on which the solutions satisîy general analytic boundary conditions.

2. As stated in the proposal for the grant, I wanted to investigate the reflection laws of elliptic equations of the form

$$
\sum_{j+k=2 n} a_{j k} D_{x}^{j} D_{y}^{k} u=0, \quad a_{j k}=\text { cons tant }
$$

across an analytic arc on which the solution $u$ satisfles $n$ general analytic boundary conditions and apply the results to the plane theory or anisotropic elasticity. Under the grant, this investigation was successrully carried out for differential boundary conditions of the same order. In this case, it was shown that reilection reduces to quadratures. The results were applied to the rirst and second fundamental boundary value problems of the plane theory of anisotropic elasticity. The enclosed paper, "Reflection Laws of High Order Elliptic Differential 
Equations in Two Independent Variables with Constant Coefficients and Unequal Characteristics Across Analytic Boundary Conditions", which has been submitted for publication, gives the results of this investigation.

\section{Work in progress.}

1. Under the grant, one research assistant and I have been working with the Culler-Fried on-line computer to try to determine ways of numerically solving non-linear singular integral equations of the form that was investigated in [20], $\mathbf{v}$. -equations of the form:

$$
w(z)=B(z)+\lambda C(w)+\lambda \vec{F}[z, w(z), \lambda]+\frac{\tilde{\lambda}}{2 \pi} \int_{\Gamma} \frac{N[t, z, w(t), \lambda]}{t-z} d t,
$$

where $z$ belongs to the closed smooth contour $\Gamma$ and the Cauchy principal value is meant by the integral, $C(w)$ is a bounded functional, $B, F$, and $\mathbf{N}$ are complex-valued functions defined for $\mathbf{z}$ on $\Gamma$ and $w$ is in some closed convex set. We were led to an equation of this form when we were trying to determine the deformation of an elastic circular cylinder in a two dimensional ideal fluid flow. I have also been led to another non-linear singular integral equation, as well as systems of non-linear singular integral equations, when trying to determine the deformation of a clrcular cylinder in an electric field. The existence and uniqueness by successive approximations given in [1] depended on $|\lambda|<\lambda_{0}, \lambda_{0}$ being specified in terms of Hiblder constants and easily determined bound constants for $B, C, F, N$, and $\Gamma$. There is no assurance that the $\lambda_{0}$ of [1] is the best choice. It is of interest to determine the largest possible value of $\lambda$ for which we have a solution, as $\lambda$ has a 
physical significance in the hydroelastic and the electrostriction problems. For the hydroelastic problem, $\lambda$ is proportionel to the uniform fluid velocity at infintty and for the electrostriction problem, $\lambda$ is proportional to the uniform electric field in which the dielectric is placed.

By using the examples of non-linear singular integral equations (n.l.s.i.e.) from hydroelasticlty and electrostriction, we hope to vary $\lambda$ and determine, in a numerically experimental way, whether the bound given in [1] is unnecessarily small. The Culler-Fried on-line computer is particularly well suited for this since:

(1) It accepts very sophisticated instructions and

(2) it displays the results on an oscilloscope.

In the bydroelastic and electrostrictive problems, the solution $w(z)$ is the conformal map of the boundary of the undeformed region onto the boundary of the deformed region. Thus the scope will display the curve which should be the cross section of the distorted cylinder. We intend to observe this distortion curve as a function of $\lambda$ (fluid velocity, field strength) by solving the n.I.s.i.e. by successive approximations. It is expected that, as $\lambda$ increases, we shall observe a breakdown, 1.e. a critical $\lambda$. This experimentally determined critical $\lambda$, if of larger value than the prescribed bound of $\lambda_{0}$ of $[1]$, would be the starting point of rurther theoretical investigation, e.g., we would try to extract those mathematical properties of the kernel arising in the physical problem, which would permit convergence for $\lambda$ 's larger than those restricted in [1], i.e., we would try to formulate an appropriate mathematical question viz. What class of non-linear singular integral equations, 
examples of which are the equations arising from tydroelasticity and electrostriction, should oe studied?

2. Under the grant I have been working with a second research assitant on the reduction $0 i$ the electrostriction problem arising by placing a spherical membrane shell in an electric rield. In this connection we hope to use the representations of the solution oi the theory of elastic spherical shells as deduced oy Vekua [2]. The research assistant has written out a translation of [2] into English.

[1] Finnila R, Sloss, J. M., A class of non-linear singular integral equations with a hydroelastic application. To appear in J. of Math. and Mech. in January or February 1967.

[2] Vekua, I. N., Integrating the Equations of Spherical Shells, Prikl. Mat. Mekh., Akademiya NAUK S.S.S.R., vol. 9, No. 3, 194.5, pp. 368-388 (Russian). 